\title{
Reliability Improvement and Risk Reduction through Self-reinforcement
}

\author{
Michael Todinov \\ Oxford Brookes University \\ School of Engineering, Computing and Mathematics \\ Oxford, Wheatley, OX331HX, UK \\ mtodinov@brookes.ac.uk
}

\begin{abstract}
The method of self-reinforcement has been introduced as a domain-independent method for improving reliability and reducing risk. A key feature of self-reinforcement is that increasing the external/internal forces intensifies the system's response against these forces. As a result, the driving net force towards precipitating failure is reduced. In many cases, the self-reinforcement mechanisms achieve remarkable reliability increase at no extra cost.

Two principal ways of self-reinforcement have been identified: reinforcement by capturing a proportional compensating factor and reinforcement by using feedback loops.

Mechanisms of transforming forces and motion into self-reinforcing response have been introduced and demonstrated through appropriate examples. Mechanisms achieving selfreinforcement response by self-aligning, self-anchoring and modified geometry have also been introduced.

For the first time, the potential of positive feedback loops to achieve self-reinforcement and risk reduction was demonstrated. In this respect, it is shown that self-energizing, fast growth and fast transition provided by positive feedback loops can be used with success for achieving reliability improvement.

Finally, a classification was proposed of methods and techniques for reliability improvement and risk reduction based on the method of self-reinforcement.
\end{abstract}

Keywords: domain-independent, reliability improvement, risk reduction, self-reinforcement, positive feedback loop

\section{Introduction}

While a great deal of agreement exists regarding the necessary steps of risk assessment, there is profound lack of understanding and insight regarding the general methods for risk reduction. The common approach to risk reduction is the domain-specific approach which relies heavily on root cause analysis and detailed knowledge from the specific application domain. Measures specific to the particular application domain are selected to reduce the likelihood of failure or the consequences from failure and the risk reduction is conducted exclusively by experts in the specific application domain.

As a result, risk reduction has effectively been fragmented into risk reduction in numerous specific domains: nuclear industry, aviation, construction industry, food storage, food processing, banking, oil and gas industry, road transportation, railway transportation, marine transportation, financial industry, information technology, environmental sciences, etc. The 
list can be continued. Each of these application domains employs specific risk reduction methods.

The domain-specific approach to risk reduction created the illusion that efficient risk reduction can be delivered successfully solely by using methods offered by the specific domain without resorting to general methods for risk reduction.

As a result, many industries have been deprived from efficient risk-reducing solutions. The same mistakes are made again and again, resulting in products and processes with inferior reliability, associated with high risk of failure. Examples of such repeating mistakes are:

- Insufficient reliability built in products with very high cost of failure;

- Designing components with homogeneous properties where the stresses are clearly nonuniform;

- Creating systems with vulnerabilities where a single failure entails the collapse of the system;

- Missed opportunities to improve reliability and reduce risk at no extra cost (e.g. by a simple permutation of components with the same function and different age);

- Redundancy compromised by a common cause.

The weaknesses of risk reduction solely based on methods from a specific domain is constantly exposed by a string of costly failures and disasters (e.g. catastrophic oil spills, financial crises, serious industrial accidents, transportation accidents, power blackouts, etc.).

In some cases, correct solutions are found but only through reinventing the wheel", after many costly and time consuming trials and errors.

An important contributing reason for this highly undesirable situation is the absence of a framework of domain-independent methods for reliability improvement and risk reduction that could provide valuable methodological knowledge to many unrelated domains. The domain-independent methods for reliability improvement and risk reduction enhance the reliability of products and operations for any company and organisation. To any company, reliability is one of the most important attributes of its products.

With the exception of a few well-known domain-independent methods for risk reduction such as: implementing redundancy, strengthening weak links, upgrading with more reliable components, simplification of components, systems and operations and condition monitoring, the framework of the domain-independent methods for reliability improvement and risk reduction is missing.

French (1999) formulated a number of general principles to be followed in conceptual design, but they were not oriented towards improving reliability and reducing technical risk. General principles to be followed in engineering design have also been discussed in Pahl (2007). However, most of the discussed principles are either not related to reducing the risk of failure or are too specific (e.g. the principle of thermal design), with no general validity. Collins (2003) discussed engineering design with failure prevention perspective. However, no reliability improvement and risk reducing principles with general validity were formulated.

The present paper contributes an important domain-independent reliability improvement and risk reduction method, referred to as the method of self-reinforcement'. A key feature of self-reinforcement is that increasing the external/internal forces intensifies the system's response against these forces. As a result, the driving net force towards precipitating failure is decreased. Self-reinforcement increases the ability of the system to absorb overloading thereby increasing the resilience of the system. As it will be demonstrated later, in many cases, the self-reinforcement mechanisms achieve a remarkable reliability increase at no extra cost.

Despite the substantial amount of existing research on improving reliability by reinforcement" of components and systems, to the best of our knowledge, no research exists 
on -self-reinforcement". However, there is a key difference between improving reliability by _reinforcement" and by _self-reinforcement". An important feature of self-reinforcement which distinguishes it from the common = 1einforcement ${ }^{6}$ is that increasing the external/internal force magnitudes increases the resistance against this force. For the common reinforcement solutions, such an increase of the system's resistance is absent. The 'reinforcement' only provides compensation against an undesirable effect but not a proportional compensation. Shot-peening of the surface of automotive suspension springs, for example, is an example of reinforcement. It introduces compressive residual stresses in the surface of the spring coil and strengthens the resistance of a loaded spring against fatigue failure (Niku Lari, 1981; Bird and Saynor, 1984). The cold expansion, used in aviation for creating compressive stresses at the surface of fastener holes (Fu et al, 2015) is another example of reinforcement. Cold expansion is done by passing a tapered mandrel through the hole. The state created in the vicinity of the hole (compressive residual stress field), counters the tensile loading stresses during operation and impedes the formation of fatigue cracks at the edge of the hole and their propagation which reduces the risk of fatigue failure.

However, increasing the load on a shot peened spring or the loading stress in the vicinity of a fastener hole, does not change the magnitude of the compressive residual stresses and does not increase the fatigue resistance of the components. Reinforcement from shot peening and cold expansion is present but not a self-reinforcement.

Mechanisms that are in fact only reinforcement mechanisms should not be confused with =self-reinforcement" mechanisms. The focus of this paper is on self-reinforcement mechanisms for improving reliability and reducing risk.

Isolated self-reinforcement solutions for improving the performance of components and systems do exist in the engineering design literature. Thus, a self-reinforcing effect from capturing rotational motion has been discussed for example in (Pahl et al., 2007). Pahl et al (2007) however, did not go beyond this isolated example and generalise the selfreinforcement method as a domain-independent method for improving the reliability of engineering assemblies and systems.

Self-locking devices, such as self-locking screws, self-locking grips, self-locking hooks, self-energizing breaks, etc., have been known for a long period of time. Costache et al (2016), for example, recently introduced self-locking grips for anchoring fibre-reinforced tendons. However, self-locking has not been recognised as a self-reinforcement method for improving reliability.

Self-balancing can also be a source of self-reinforcing response. The _Roly-Poly' toy which rights itself when pushed can be given as an example. Recently, self-balancing inspired by the =Roly-Poly' toy has been suggested by Zhang et al (2013) for improving the side rolling stability of an e-bike. Again, self-balancing has not been recognised as a method for improving reliability by self-reinforcement.

Eliminating harmful factors and influences is the purpose of many inventions and Altshuller's TRIZ system (Altshuller, 1984,1996, 1999) captured a number of useful general design principles that could be used to eliminate harm and reduce risk. No discussion however has been presented by Altshuller related to improving reliability by selfreinforcement. No discussion regarding improving reliability by self-reinforcement has been presented in more recent literature related to TRIZ (Terninko et al. 1998; Savransky 2000; Orloff 2006; Rantanen and Domb, 2008; Gadd 2011).

In summary, despite the availability of occasional isolated self-reinforcement solutions used in engineering designs, they were not recognised as instances of the method of selfreinforcement and were not linked to this method. No analysis of the mechanisms through which self-reinforcement improves reliability and reduces risk or a classification of selfreinforcement mechanisms has ever been presented in the engineering design literature or the 
reliability and risk literature. Consequently, this paper attempts to fill this gap by providing an introduction to the mechanisms through which the method of self-reinforcement improves reliability and reduces risk.

The contribution of this paper consists of identifying and introducing for the first time the two principal ways of self-reinforcement: self-reinforcement by capturing a proportional compensating factor and reinforcement by using feedback loops.

Another contribution of the paper is the introduction of positive feedback loops as a risk reduction resource. In this respect it is shown that the self-energising, the fast growth and fast transition provided by the positive feedback loops are important mechanisms for achieving self-reinforcement response.

The paper also identified the following basic mechanisms of self-reinforcement by capturing a proportional compensating factor: (i) mechanisms transforming forces and pressure into a self-reinforcing response; (ii) mechanisms transforming motion into a selfreinforcing response; (iii) mechanisms achieving a self-reinforcing response by modifying geometry or the development of strains (iv) mechanisms achieving self-reinforcement response by self-aligning.

The classification of the self-reinforcement mechanisms presented in this paper is based on a number of solutions, from various engineering fields. Each of the available solutions was analysed to verify and, in some cases, to quantify the effect of self-reinforcement on the reliability improvement and risk reduction. The available solutions were also analysed for recurring patterns and invariants. A certain level of abstraction was used to strip the available solutions from their specific engineering context and uncover the underlying act of selfreinforcement.

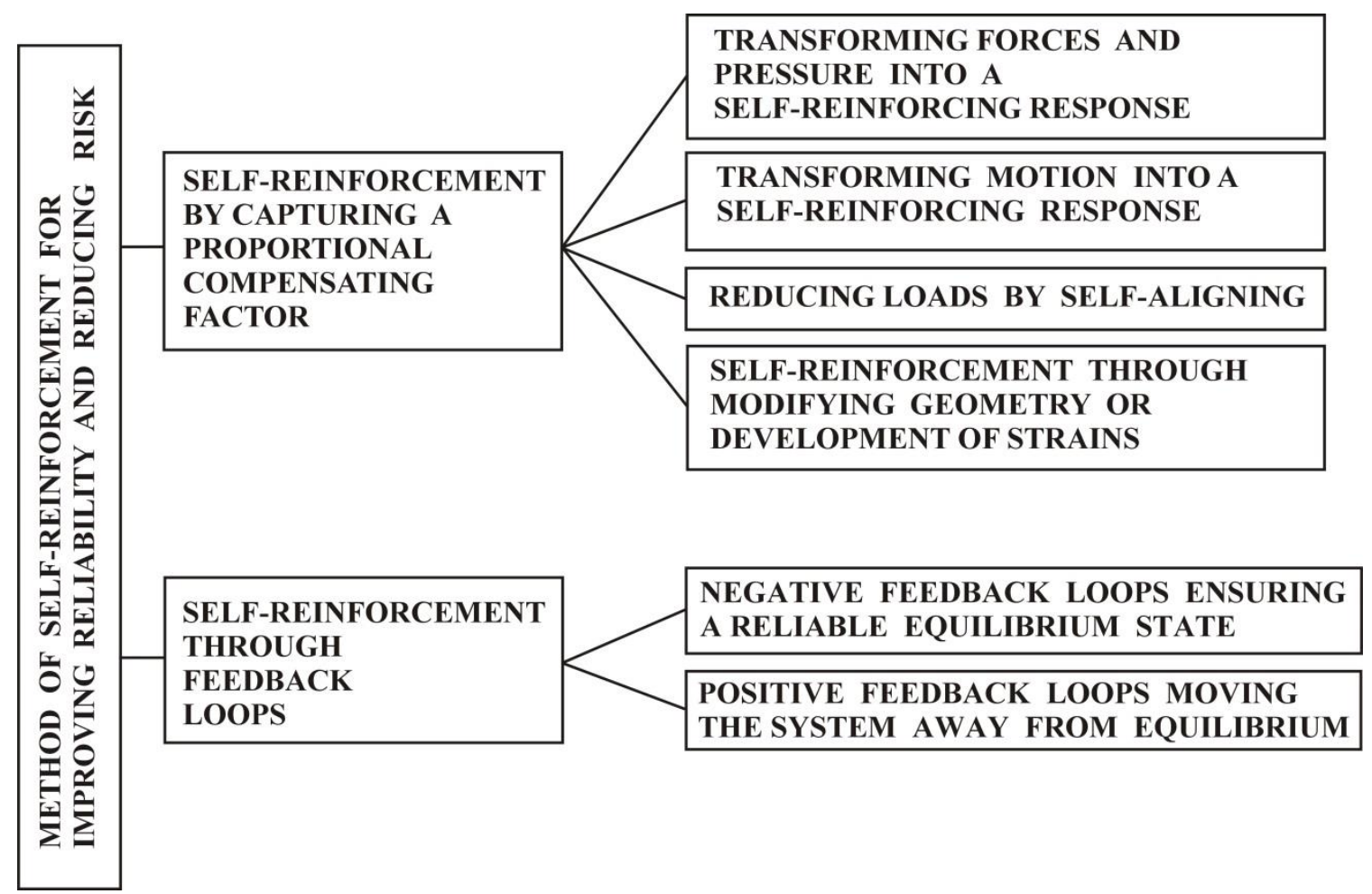

Figure 1 The method of self-reinforcement for improving reliability and reducing risk.

From the large body of available solutions, the emerging patterns were captured and distilled into distinct categories. A classification summarising these categories, classes and techniques has been presented in Figure 1. In what follows, the identified mechanisms of reliability improvement and risk reduction by self-reinforcement are discussed in detail. 


\section{Self-reinforcement relying on a proportional compensating factor}

Self-reinforcement by a proportional compensating factor is present in cases where a particular effect is captured and used to provide a proportional, compensating, selfstrengthening response. The compensating response is channelled towards reducing the driving forces precipitating failure.

\subsection{Transforming forces and pressure into a self-reinforcing response}

\subsubsection{Capturing a self-reinforcing proportional response from friction forces}

Friction forces can be captured and used as self-reinforcing counterforces. Common applications are the self-locking screws and self-locking wedges. The higher the load in selflocking devices, the higher is the magnitude of the excited friction forces, the larger is the reliability of the self-locking device. Self-reinforcement is also found in: (i) self-locking grips in tensile testing machines; (ii) self-locking plate clamps; (iii) self-locking hooks; (iv) selflocking climbing equipment, (v) self-locking marine cleats, etc.

Consider the block in Figure 2a loaded with the external force $P$ and experiencing friction. Increasing the external force $P$ increases proportionally the horizontal component $P_{x}$ (undesirable outcome $B$ ) working towards disturbing the equilibrium of the body (Figure 2a). It is a well-documented fact from statics (e.g. Meriam et al. 2016) that for the simple system in Figure 2a, no matter how large the loading force $P$ is, if it lies within the friction cone defined by the friction angle $\rho(\mu=\tan \rho$, where $\mu$ is the static coefficient of friction), the body will remain in a state of equilibrium. Increasing the magnitude of the loading force $P$ increases the component $P_{x}$ pushing the component along the horizontal axis but also increases the normal force $N=P_{y}$ which excites the friction force $F$. The friction force $F$ is proportional to the normal force $N, F=\mu N$ and can be interpreted as the third factor $C$, whose magnitude is increasing with increasing the magnitude of the undesirable component $P_{x}$ (Figure $2 \mathrm{~b}$ ) and is subtracted from the output (the component $P_{x}$ ). The friction force $F$ fully compensates the undesirable force $P_{x}$. The body is in equilibrium. Self-reinforcement effect is present because with increasing the external load $P$, the resistance against moving along the horizontal axis $x$ also increases.

The essence of the mechanism of self-reinforcement by capturing a compensating proportional response has been captured with the abstract diagram in Figure 2b.

(a)

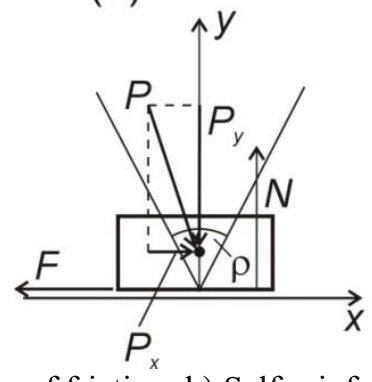

(b)

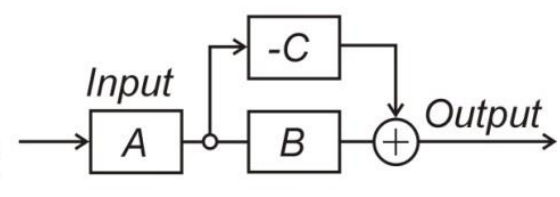

Figure 2 a) A cone of friction; b) Self-reinforcement by capturing a compensating third factor $C$ whose magnitude is proportional to the undesirable outcome $B$

Factor $A$ results in a undesirable outcome $B$. By an appropriate modification (or directly), the effect $C$ is captured to create a compensating response, whose magnitude is proportional 
to the magnitude of the undesirable outcome $B$ but whose sign is opposite. Now, if the compensating effect $C$ is added to outcome $B$, the resultant undesirable outcome will be partially or fully cancelled. This is a self-reinforcement effect because with increasing the magnitude of the undesirable outcome $B$, the magnitude of the compensating effect $C$ also increases. As a result, the driving force towards failure precipitation is reduced and reliability is improved. The self-reinforcement by capturing a compensating effect increases the capability of the system to absorb overloading shocks which improves the resilience of the system.

2.1.2 Case study: Transforming friction forces into a proportional response in the design of a friction grip

Self-reinforcement by capturing a proportional compensating factor has been used in the design of a friction grip (Figure 3).

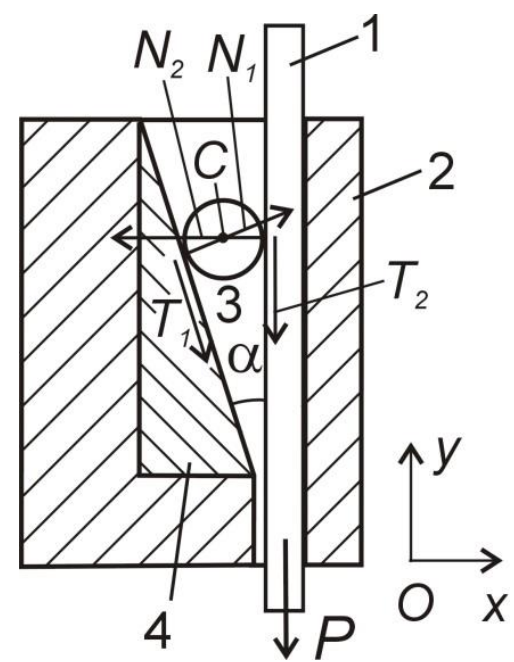

Figure 3 A self-reinforcing design of a friction grip based on exciting compensating friction forces.

The friction grip includes the strip 1 which is being pulled downwards with a force $\mathrm{P}$, the block 2, cylinder 3 and wedge 4 . The static friction coefficient between the cylinder 3 and the wedge 4 is $\mu_{1}$; the friction coefficient between the cylinder 3 and the strip 1 is $\mu_{2}$. The forces acting on the cylinder 3 are: the normal force $N_{1}$ and the friction force $T_{1}=\mu_{1} N_{1}$ from the wedge 4 , the normal force $N_{2}$ and the friction force $T_{2}=\mu_{2} N_{2}$ from the strip 1 . The cylinder 3 has a radius $r$ and its weight has been ignored as being too small compared to the rest of the forces.

Under certain conditions, self-reinforcement will be present in the system because increasing the external load $P$ will increase the friction forces $T_{2}$ and $T_{1}$ (factor $C$ from Figure $2 b$ ) which retain the strip and cylinder in equilibrium.

To determine the conditions under which self-reinforcement is present, the equilibrium conditions for cylinder 3 are considered. They include three equations: the sum of the projections of the forces along the $x$-axis must equal zero; the sum of the projections of the forces along the $y$-axis must equal zero and the sum of the moments of the forces with respect to the centre $C$ of the cylinder must also equal zero:

$$
\begin{aligned}
& N_{1} \cos \alpha+\mu_{1} N_{1} \sin \alpha-N_{2}=0 \\
& N_{1} \sin \alpha-\mu_{1} N_{1} \cos \alpha-\mu_{2} N_{2}=0 \\
& \mu_{1} N_{1} r-\mu_{2} N_{2} r=0
\end{aligned}
$$


In equation (3), $r$ is the radius of cylinder 3.

From equation (3), $N_{2}=\frac{\mu_{1}}{\mu_{2}} N_{1}$. Substituting $N_{2}=\frac{\mu_{1}}{\mu_{2}} N_{1}$ in equations (1) and (2), followed by dividing the equations by $N_{1}$ (assuming that $N_{1}>0$ ) results in:

$$
\begin{aligned}
& \cos \alpha+\mu_{1} \sin \alpha-\frac{\mu_{1}}{\mu_{2}}=0 \\
& \sin \alpha-\mu_{1}(1+\cos \alpha)=0
\end{aligned}
$$

From equation (5), $\mu_{1}=\frac{\sin \alpha}{1+\cos \alpha}=\frac{2 \sin (\alpha / 2) \cos (\alpha / 2)}{2 \cos ^{2}(\alpha / 2)}=\tan (\alpha / 2)$. From equation (4), $\mu_{2}=\frac{\mu_{1}}{\cos \alpha+\mu_{1} \sin \alpha}$.

Self-reinforcement (equilibrium of the cylinder 3) will only be present if, for the coefficients of friction, the following inequalities are simultaneously fulfilled:

$$
\begin{gathered}
\mu_{1} \geq \tan (\alpha / 2) \\
\mu_{2} \geq \frac{\mu_{1}}{\cos \alpha+\mu_{1} \sin \alpha}
\end{gathered}
$$

If the two coefficients of friction are equal, equation (6) is obtained as a condition for selfreinforcement.

In this case study, the reliability improvement through self-reinforcement has been obtained at no extra cost.

\subsubsection{Transforming pressure into a self-reinforcing response}

This self-reinforcement mechanism is present when external/internal forces or states are channelled towards inducing counterforces which strengthen proportionally the system's response. Such self-reinforcement mechanism is illustrated in the next figure, in the design of a cover for containers under pressure (Figure 4).

(a)

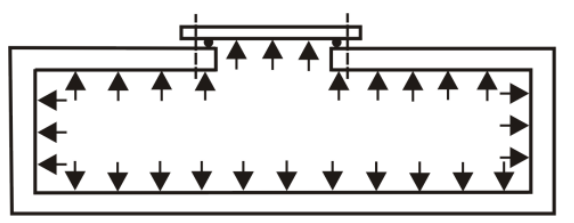

(b)

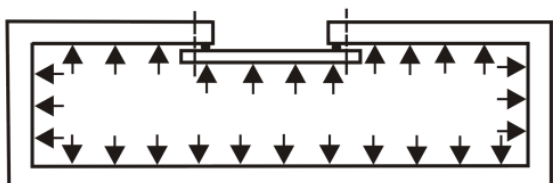

Figure 4. Improving reliability through self-reinforcement by changing the relative position of the cover and container.

For the design in Figure 4a, the loading stresses excited in the screws holding the cover can be reduced and the reliability of the seal increased significantly by changing the position of the cover with respect to the pressure vessel. The design from Figure $4 \mathrm{~b}$ channels the force induced by the internal pressure into a self-reinforcing response which helps to form a reliable seal and prevent leakage. Increasing pressure increases the magnitude of the selfreinforcing response, strengthens the seal and increases the resistance to the increased pressure. In this example, reliability has also been improved at no extra cost by applying the method of self-reinforcement.

A similar effect is present for injection pistons with self-reinforcing seals. The elastic material of the seal is pressed against the walls of the cylinder. The higher the pressure, the 
tighter the seal formed between the piston and the cylinder. Reliability improvement is obtained at no extra cost.

\subsubsection{Transforming moments into a self-reinforcing response}

Self-reinforcement by capturing a factor providing a compensating proportional response can also be illustrated by an example related to capturing a counter-moment as a proportional compensating factor (Figure 5). Figure 5a features the overturning moment on a wall resisting the pressure from a large mass of liquid or granulated substance (water, concrete, sand, etc.). If an extension is firmly fixed to the lower end of the wall (Figure 5b), the distributed weight $G$ of the mass of the liquid (granulated substance) will create an opposing moment (factor $C$ in Figure 2b) which counteracts the overturning moment of the force $P$ (factor $B$ in Figure $2 \mathrm{~b}$ ) The result is a reduced total overturning moment and a reduced risk of overturning the wall. Increasing the mass of the liquid (granulated substance) increases the overturning moment but also increases the opposing moment from the weight $G$.

(a)

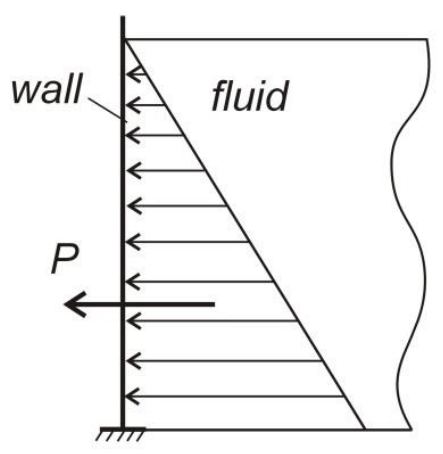

(b)



(c)

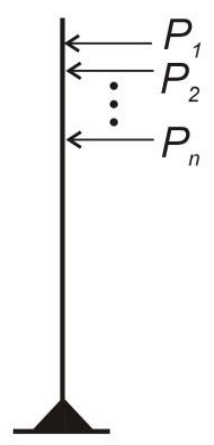

(d)

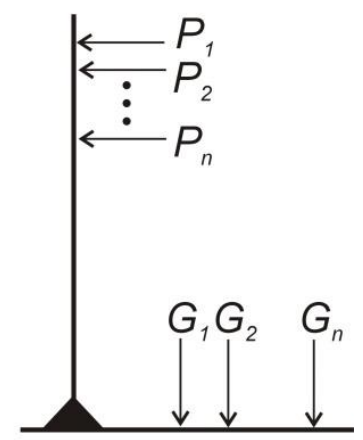

Figure $5(a, b)$ Reducing the risk of overturning of a wall supporting liquid or granulated substance by selfreinforcement. (c,d) Reducing the risk of overturning of a crowd fence by self-reinforcement.

The solution related to reducing the risk of overturning in Figure $5 \mathrm{~b}$ can be replicated to reduce the risk of overturning of crowd fences. A fence without a self-reinforcement (Figure $5 \mathrm{c}$ ) can be overturned relatively easily by the forces $P_{i}$ created by people pushing the fence (Figure 5c). If the lower end of the fence on the crowd side is made wider (Figure 5d), people will have to stand on the fence while they push against it. As a result, the distributed weight of the people (the forces $G_{i}$ in Figure 5d) standing on the lower part of the fence create an opposing moment that counteracts the overturning moment of forces $P_{i}$ from the people pushing the fence. The more people push on the fence, the more weight forces $G_{i}$ will be available for counteracting the overturning moment. The weight of people is effectively channelled towards self-reinforcement. The capability of the system to resist and absorb overloading is increased which results in improved resilience. The reliability improvement in these examples is obtained at a low cost.

\subsubsection{Self-reinforcement by self-balancing}

Unbalanced forces cause premature wear out, fatigue degradation and failure. As a rule, improving the level of balancing in a system improves the uniformity of the load distribution, reduces the magnitudes of inertia forces and loading stresses and increases the reliability of components and systems. 
Self-balancing improves the capability of the system to adapt to adversity and recover, which enhances the resilience of the system.

Self-balancing is often achieved through symmetrical design and eliminates unwanted inertia forces and moments in rotating machinery. Static balancing for example, guarantees that the mass centre of the rotating mass is on the rotation axis (Uicker, 2003). Increasing the speed of rotation increases the unbalanced inertia force but also increases the counterbalancing force. The result is a self-strengthening response.

Self-reinforcement, by generating a similar in magnitude and opposite in direction counterforce that subtracts from the force creating the undesirable effect, is at the heart of eliminating or reducing end-thrust forces. Force balancing can be done by a symmetrical design and used to minimise the axial forces on turbine shafts (Matthews, 1998). For the design in Figure 6a, the axial force $F$ from the turbine 1 needs to be counterbalanced by the thrust bearing force $F$ ' from the thrust bearing 2. For the symmetrical design in Figure 6b, the increase of the axial force $F$ from turbine 2, is accompanied by a similar increase of the equal and opposite force $F$ ' from turbine 1. The two forces counterbalance and the thrust bearing $z$ is no longer needed. The reliability of the assembly has been improved.

A sudden increase of the force $F$ will cause an equal and opposite force $F^{\prime}$ counterbalancing the increase of the loading force $F$. As a result, the capability of the system to absorb overloading shocks is improved thereby improving the resilience of the assembly.

(a)

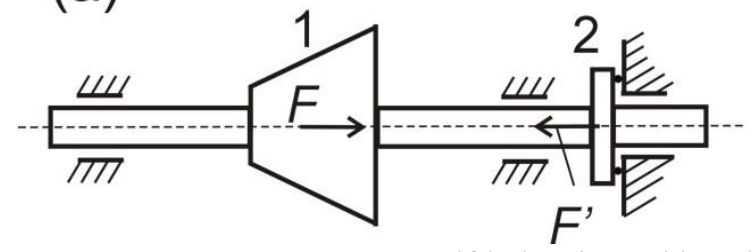

(b)

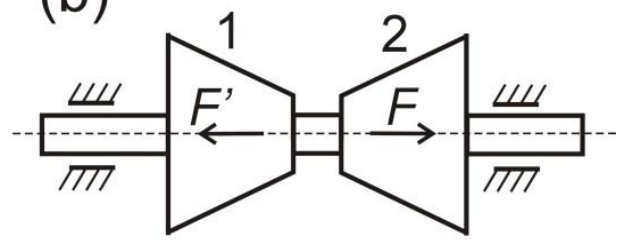

Figure 6. Self-balancing achieved through symmetrical design

The herringbone gears provide another example of self-reinforcement by counterbalancing. The axial forces in herringbone gear meshing are counterbalanced, which eliminates the need for thrust bearings. An increase in the transmitted torque increases simultaneously the magnitude of each axial force and because they act in opposite directions, the result is a very small resultant axial force.

Another example of self-balancing achieved through symmetrical design can be found in symmetrical epicyclic gear mechanisms where self-balancing of the radial forces acting on the central shaft is present.

Twisting wires to cancel their magnetic interference is also an example of selfreinforcement by self-balancing (Figure 7a). The flow of current through the wire results in electromagnetic field around the wire which could generate noise in the neighbouring wires. Twisted wires carry equal and opposite currents whose electromagnetic fields cancel. 
(a)
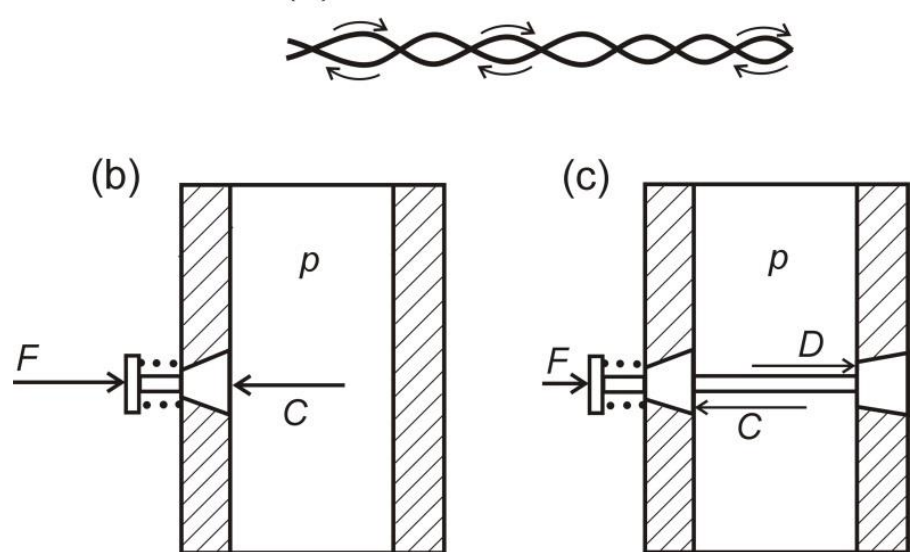

Figure 7. Other cases of self-reinforcement by self-balancing.

Finally, self-balancing can be implemented to reduce the magnitude of required activation forces if a very fast response is needed. The pipe in Figure $7 \mathrm{~b}$ is under pressure $p$. If pressure needs to be released quickly, an actuation force $F$ must be applied to the valve. The actuation force $F$ must overcome not only the resistance of the spring but also the force $C$ due to the pressure $p$ (Figure 7b). In the self-reinforcing design in Figure 7c, a counterbalance force $D$ is present. To release the pressure, the actuation force $F$ must now overcome only the resistance of the spring and a small resultant force $C-D$. The required actuation force $F$ for the design in Figure $7 \mathrm{c}$ is significantly smaller than that for the design from Figure $7 \mathrm{~b}$ and the quick pressure release is more reliable. Even in the presence of a very large pressure $p$, the valve will still be released with a relatively small force $F$. Increasing pressure $p$ increases the pressure force $C$ but also proportionally increases the counterforce $D$. A self-reinforcing effect is present.

\subsubsection{Self-reinforcement by self-anchoring}

Self-reinforcement by self-anchoring is present in cases where the loading forces excite counterforces which oppose the loading forces. This results in a reduced driving force for failure initiation. Self-anchoring bolts and screws are often used as a secure attachment to walls. Increasing the pulling force increases the anchoring effect and the pull out resistance.

Consider the cantilever double-beam structure in Figure 8a including two short links with a large cross section. The loading force $F$ excites a moment $F \times d$ and friction forces which retain the beams in the wall.

If the excited friction forces are insufficient to retain the links, the links will be pulled out and cause structure collapse.

In Figure $8 \mathrm{~b}$, in addition to the friction forces, the loading force $F$ excites additional anchoring forces $C$ in the supports. Increasing the magnitude of the loading force $F$ increases the magnitude of the anchoring forces $C$. Because of the existing self-anchoring effect, the assembly in Figure $8 \mathrm{~b}$ is more resistant against collapse compared to the assembly in Figure 8a. The short lengths of the links and the large cross sections exclude buckling as a failure mode. 
(a)

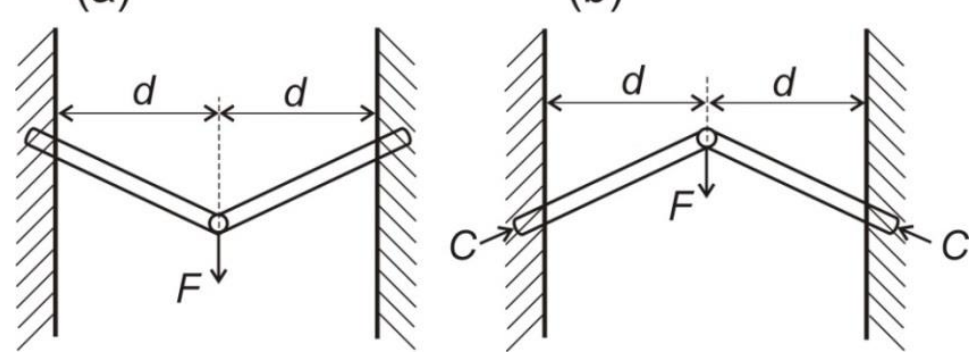

Figure 8 Self-reinforcement by self-anchoring. (b)

Consider the device in Figure 9 designed for lifting steel drums 4 with mass $m$. The U-shaped brackets 1 connect the lifting ring 3 with the hooks 2 . The device hooks over the end lips of the drum. From the equilibrium condition of the forces: $2 \times 2 T \times \cos \beta=m g$, the tension force $T$ acting in each rod of the U-shaped bracket can be determined: $T=\frac{m g}{4 \cos \beta}$. The retaining force $R$ with which the hook presses on the drum (Figure 9) can be approximated by $R=2 T \times \sin \beta=\frac{m g}{2} \tan \beta$. With increasing the weight of the drum $m g$, the retaining force $R$ also increases. Self-reinforcement is present. Increasing the angle $\beta$ towards $90^{\circ}$ results in a significant increase of $\tan \beta$ and the retaining force $R$.

In both cases, the reliability improvement by self-anchoring is obtained at no extra cost.

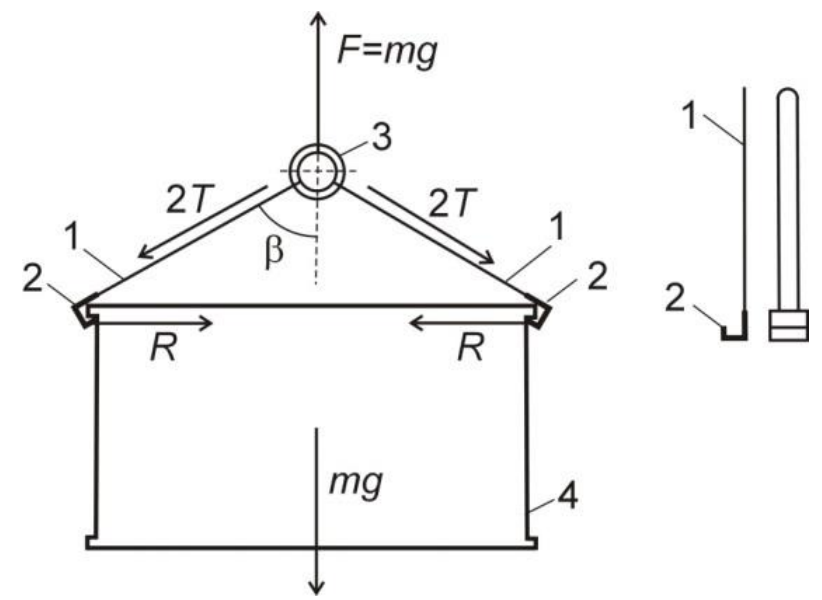

Figure 9 Hoisting a steel drum by self-reinforcement achieved through self-anchoring.

\subsection{Transforming motion into a self-reinforcing response}

This self-reinforcement mechanism is, for example, present in racing cars where wings are used for creating an aerodynamic downforce which enhances the grip with the racing track and prevents the loss of grip at high speeds while cornering. Increasing the speed of the car increases the inertia force acting on the car moving around a corner but also proportionally increases the downforce which strengthens the grip with the racing track. This is an example of self-reinforcement obtained by capturing and using motion to provide a compensating, self-strengthening response. The higher the speed, the higher is the magnitude of the downforce, the larger is the magnitude of the self-reinforcing response.

The next self-reinforcing effect obtained from capturing rotational motion has been taken from an example discussed in (Pahl et al., 2007, Figure 10a,b). 

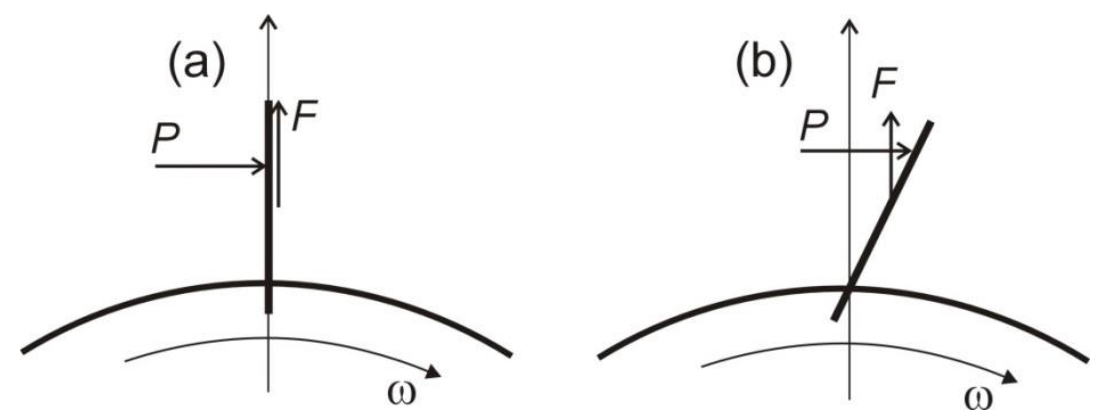

Figure 10 Reducing the stresses at the base of a blade attached to a rotor by capturing a proportional compensating moment

The bending stresses at the base of a blade due to the tangential force $P$ (undesirable outcome $B$, Figure 10a) can be reduced if the centrifugal force resulting from the rotation of the blade is channelled to create an opposing moment counteracting the moment created by the tangential force $P$. Channelling the centrifugal force to create an opposing moment (proportional compensating factor $C$ ) can be done by inclining the blades at an angle from the vertical (Figure 10b). For the design in Figure 10b, the centrifugal force $F$ from the rotation of the blade creates a proportional moment counteracting the bending moment from the tangential force $P$. This reduces the overall bending moment and the stresses at the base of the blade. The larger the tangential force $P$, the larger is the angular speed $\omega$, the larger is the centrifugal force (proportional to $\omega^{2}$ ), the larger is the counteracting bending moment created by the centrifugal force.

As a result, the capability of the assembly to absorb and withstand a sudden increase in the rotational speed is increased which improves the resilience of the assembly and reduces the risk of failure. Reliability improvement by self-reinforcement is obtained at no extra cost.

\subsection{Self-reinforcement by self-alignment}

The mechanism of self-reinforcement by self-alignment is based on using external forces to alter the position/orientation of the object which results in reduced loading stresses. Selfalignment improves the capability of the system to adapt to adverse operational conditions which enhances its resilience.

An example of a self-alignment mechanism can be given with the self-aligning ball bearing whose outer rings have spherical raceways (Bergman et al, 2009). The self-aligning ball bearing accommodates inevitable misalignments of the shaft leading to increased levels of the loading stresses. Without the benefit of the self-alignment, the life of the ball bearing will be reduced significantly.

\subsubsection{Case study: Self-reinforcement by self-alignment of a rectangular panel under wind pressure}

Self-reinforcement can also be achieved if the loading force induces self-alignment which reduces the loading force. Consider a common case where the surface of a rectangular panel with side lengths $L$ and $M$ is perpendicular to the wind (Figure 11a). In this case, the dynamic pressure of the wind $(1 / 2) \rho_{a} v_{a}^{2}$ is transformed into a force $F_{w}=C_{d}(1 / 2) \rho_{a} v_{a}^{2} A_{p}$ acting on the panel, where $C_{d}$ is the drag coefficient characterising the panel, $\rho_{a}=1.2$ is the mass density of air, $v_{a}$ is the air velocity and $A_{p}=L \times M$ is the projected surface area of the panel perpendicular to the velocity of the wind. The wind force $F_{w}$ can reach a large magnitude because it increases with the square of air velocity. Thus, wind with velocity $v_{a}$, 
perpendicular to the panel, whose mass centre $G$ is at a height $h$ above the ground creates the force $F_{w}=(1 / 2) \times C_{d} \times \rho_{a} \times v_{a}^{2} \times A_{p}$ and the moment

$$
M_{w}=F_{w} h=(1 / 2) \times C_{d} \times \rho_{a} \times v_{a}^{2} \times A_{p} \times h
$$

This bending moment could reach significant magnitude which will result in significant bending stresses at the base of the support.

However, if the panel is made to rotate about an axis (point $O$ in Figure 11b) which is off the axis going through the mass centre $G$, the wind pressure will rotate the panel until the resisting moment counterbalances the moment rotating the panel. Suppose that this balancing occurs at an angle $\alpha$. In this case, the projected area is $A_{p}=L \times M \times \cos \alpha$ which results in bending moment $M_{w}$ with a smaller magnitude.

(a)

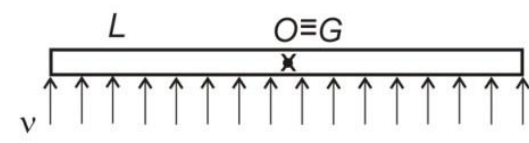

(b)

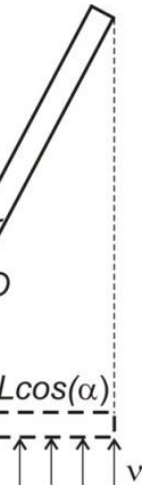

Figure 11. Self-reinforcement by self-alignment (reducing the stresses at the base of a square panel with length $L$ and height $M$ ).

Increasing the velocity of the wind produces a self-reinforcing response. Indeed, increasing the air velocity $v_{a}$ increases the angle $\alpha$ at which the resisting moment counterbalances the moment rotating the panel. Increasing the magnitude of $\alpha$ reduces the projected area $A_{p}=L \times M \times \cos \alpha$ which causes a corresponding reduction of the bending moment $M_{w}$ at the base of the support. In other words, increasing the wind velocity intensifies the system's response against the increased wind velocity. As a result, the capability of the assembly to absorb overload and adapt to adverse operational conditions is improved which enhances the resilience of the assembly. The reliability improvement has been obtained at no extra cost.

The principle behind this self-reinforcing design can even be used in household items like umbrellas. If an umbrella, held against the wind, is made to change its area by partially collapsing, an increase in the strength of the wind will reduce the exposed surface of the umbrella. This in turn will reduce the wind force on the umbrella and will result in reduced loading stress and increased reliability.

\subsection{Self-reinforcement through modified geometry and strains}

Modifying the geometry of a loaded component is often associated with a more uniform load distribution which decreases the stresses in the material and results in improved loadbearing capacity. Such are for example the nuts with special geometry that are associated with more even distribution of the load and higher load-carrying capacity of the thread (Matthews, 1998). The higher the load, the more uniform the load distribution along the thread. Making the shape of a component symmetric often brings counterbalancing forces (Matthews, 1998) which increase the load-bearing capacity. 
In materials, a self-reinforcing effect is present during plastic deformation which is known as strain hardening (Dowling, 1999). The resistance of the material to plastic deformation (the self-reinforcing effect) increases with increasing the deformation strains.

Another example of self-reinforcement by strains can be found in the strain hardening of Hadfield manganese steel (Lindroos et al, 2015). Increasing the impact loading improves the resistance of the steel to abrasive wear. A similar self-reinforcing effect is present in cubic zirconia ceramic with dispersed tetragonal zirconia particles (Kelly and Francis-Rose, 2002). For an existing crack in the ceramic material, increasing the crack tip opening load intensifies the stress field around the tip of the crack. The intensified stress field triggers the transformation of the embedded tetragonal zirconia particles into monoclinic zirconia which has a larger specific volume. The volume expansion associated with the phase transformation strains exerts a crack-closing force on the tip of the crack which resists crack propagation. These are examples of self-reinforcement resulting from phase transformation strains.

\section{Self-reinforcement by feedback loops}

\subsection{Self-reinforcement by creating negative feedback loops}

This mechanism utilizes negative feedback control and achieves self-reinforcement and reliability improvement through the process described next. The feedback loop is a circular chain of causes and effects. The negative feedback loop seeks a goal around which the system is stabilised. The negative feedback samples the output of the system and information about the deviation of the output from the goal is used to control the input in such a way that the deviation is diminished. If the output signal is above the goal, the negative feedback loop corrects the input signal in such a way that the output is decreased towards the goal. If the output signal is below the goal, the negative feedback loop corrects the input signal in such a way that the output is increased towards the goal (Figure 12a).

The larger the deviation of the output from the goal, the larger is the input correction counteracting the deviation of the output.

Negative feedback loops are widely used in control systems to guarantee specified position, temperature, pressure, humidity, concentration, speed of rotation, etc.

The negative feedback loop is commonly found in the coupling of an induction motor and a machine. An increase in the angular velocity of the rotor from the point of stable operation causes the driving torque to drop below the resisting torque which decreases the angular velocity of the rotor. A decrease in the angular velocity of the rotor causes the driving torque from the induction motor to increase above the resisting torque. The result is an increase of the angular velocity of the rotor.

(a)

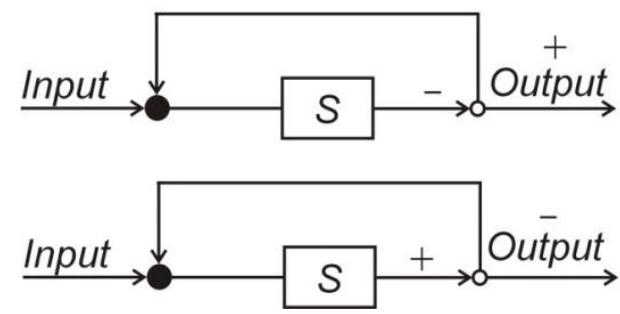

(b)

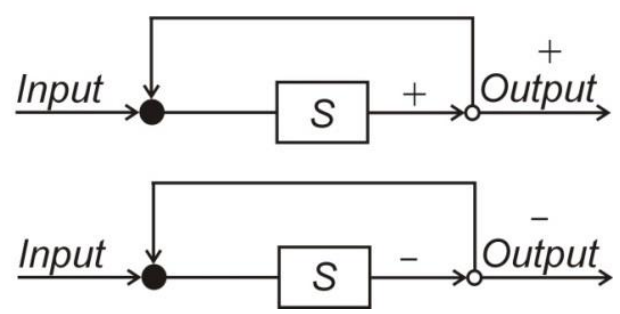

Figure 12 a) Negative and b) positive feedback loop. 
Commonly, in negative feedback control systems, the output is measured and fed back to an error detector at the input. A controller then corrects the parameters of the system/process so that the deviations from the set output become as close to zero as possible. The availability of low-cost electrical devices and sensors makes it possible to provide flexibility and regulate mechanical systems to a finer degree compared to all-mechanical systems. The negative feedback loop improves the capability of the system to recover if the balance is disturbed which results in improved system resilience.

In general, interpreting and acting upon a flow of information about the state of a system in order to stabilise its output, is effectively a negative feedback control. Whenever the information flow indicates that a particular system parameter has drifted from the set value, an action is taken to return the system towards the set value of the parameter. In this respect, measurements from sensors, together with actions following the interpretation of the measured values, is a common form of a negative feedback loop. For example, the measured speed provided by a radar speed sign helps motorists control their speed according to the permitted speed limit.

\subsection{Positive feedback loops}

While the application of negative feedback control loops is well-understood and welldocumented, there is a profound lack of discussion in the reliability and risk literature on the application of positive feedback loops to improve reliability and reduce risk.

The positive feedback loop is also a circular chain of causes and effects. The mechanism of positive feedback loops consists of correcting the input in such a way that a further deviation of the output occurs in the same direction (Figure 12b). If the output is increasing, the positive feedback loop leads to a further increase of the output. If the output is decreasing, the positive feedback loop leads to a further decrease of the output (Figure 12b). In this way, the mechanism of positive feedback loops works towards moving the system away from equilibrium. Feedback loops are presented by feedback loop diagrams (Figure 13). The links on the diagrams imply a direction of causation, and not simply a time sequence. Thus the positive link from $A$ to $B$ in Figure 13a means when $A$ increases then $B$ increases "while the negative link from $B$ to $C$ means = when $B$ increases then $\mathrm{C}$ decreases ${ }^{6}$

A positive feedback loop may not necessarily be based on a single-stage control from the output, directly to the input. A positive feedback loop could include a long chain of cause and effect relationships the final stage of which affects the input in such a way that a deviation of the output in one direction leads to further deviation of the output in the same direction. Thus, in the loop depicted in Figure 13a, increasing the input factor $A$, leads to an increase of factor $B$. The increase of factor $B$ leads to a decrease of factor $C$ which causes an increase of factor $D$. The increase of factor $D$ finally causes an increase of the input $A$. As a result, an increase of factor $A$ leads to a further increase of factor $A$.

If an initial base level exists, a slight deviation from the base level tends to produce a further deviation due to the positive self-reinforcing feedback loop. In this sense, the positive feedback loop works towards increasing the gap between the current state of the system and its base-level state. If the initial deviation is positive with respect to the base level, the positive feedback loop initiates a further positive deviation, which is a process of growth. If the initial deviation is negative, the positive feedback loop initiates a further negative deviation which is a process of decay/decline.

Positive feedback loops work towards destabilising the system's output and moving it away from equilibrium. The positive feedback loops are important reasons behind prolonged droughts, the global warming, bank runs, etc. This is why, it is widely believed that positive feedback loops are always associated with destruction and collapse. However, positive 
feedback loops are also important drivers behind growth that can be utilised for reliability improvement and risk reduction.

(a)

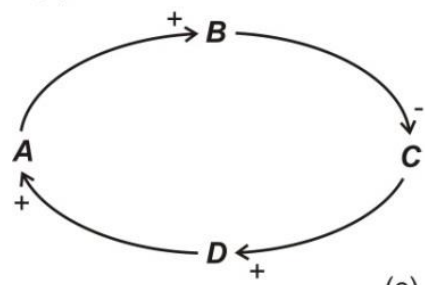

(b)

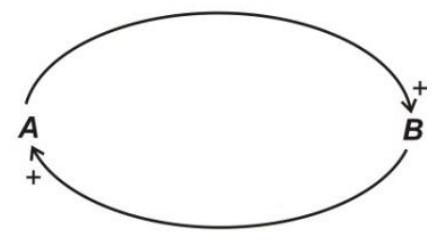

(c)

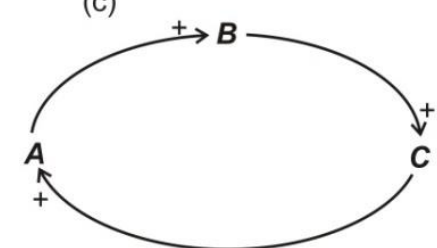

Figure 13 Positive feedback loop diagrams.

There are two principal pathways to reducing risk through positive feedback loops: (i) by eliminating or inhibiting self-reinforcing feedback loops with negative impact and (ii) by promoting positive self-reinforcing feedback loops with positive impact.

\subsection{Reducing risk by eliminating or inhibiting positive feedback loops with negative impact}

Significant risk-reduction can be achieved by discovering and eliminating positive feedback loops destabilising a system.

An example of a positive feedback loop with negative impact is the exothermal chemical reaction. The heat (cause $A$ ) released during the chemical reaction (Figure 13b), if not removed, causes the reaction to occur at a higher speed (effect $B$ ) which in turn leads to more released heat $A$, etc. This positive feedback loop could lead to a thermal runaway and chemical explosion. Such positive feedback loops are present in lithium-ion batteries used as sources of electrical power in various electronic devices and electric vehicles. The critical temperature at which the side reactions causing thermal runaway start is known as thermal runaway onset temperature. The thermal runaway onset temperature can be reached during an accident causing a short circuit in some of the battery cells, during overheating or overcharging (Feng et al, 2017). Thus, eliminating the possibility for a runway chemical reaction by redesigning the chemical process essentially reduces risk by removing positive feedback loops with negative impact.

The negative impact from positive feedback loops in lithium-ion electrical batteries due to a thermal runaway for example, can be prevented if additives are used to switch off the current at high temperatures (Feng et al, 2017). This measure effectively introduces a negative feedback loop which stabilises the system.

The negative impact from the positive reinforcing feedback loop in electrical batteries can be prevented if the positive feedback loop is inhibited by adequately cooling the battery cells so that a specified temperature of the battery cells is continuously maintained. Alternatively, the materials in the battery cell can be modified to improve their thermal stability. Reducing the likelihood of a thermal runaway can also be done by a properly designed battery management system that ensures a proper cooling of the battery cells.

It is important to note that before reaching the onset temperature at which the positive feedback loop and the thermal runaway reaction start, a negative feedback loop is in place. 
Thus, increasing the temperature of the cell leads to a larger thermal gradient which leads to a larger heat transfer and heat loss. As a result, further increase of the temperature is halted. The system is in a stable equilibrium. However, beyond a certain triggering temperature, the larger thermal gradient and heat transfer are not sufficient to stop the temperature increase of the cell which marks the start of a positive self-reinforcing feedback loop.

Interconnected networks often exhibit positive feedback loops with negative impact. In interconnected power and telecommunication networks, the telecommunication network is powered from the power network while the power network is controlled by the telecommunication network.

Failure of a number of nodes in the power network due to overloading caused by a random failure (factor $A$ in Figure 13b) causes failures of a number of nodes in the connected telecommunication network (factor $B$ in Figure 13b). Failure of nodes in the telecommunication network cause further failures in the power network and this process continues until both networks collapse. The positive feedback loop creates vulnerability that can be eliminated, for example, by decoupling the two networks in times of crisis. Decoupling destroys the positive feedback loops between the networks and brings the systems into a stable condition. Since the interdependency between systems is increasing, the importance of eliminating positive feedback loops is also increasing.

Removing positive feedback loops with negative impact due to capacitive or inductive couplings is an essential component of debugging circuits. Thus, removing positive feedback loops with negative impact from amplification circuits reduces unwanted spontaneous oscillations and greatly improves the stability of the amplifier.

Positive feedback loops in a loaded structure could cause fast deterioration and collapse. The process continues as follows. An overload causes some of the load-carrying elements of the structure to fail. The load is then redistributed and carried by a smaller number of elements. The higher load per element causes their rate of deterioration to increase which causes more of the elements to fail. This in turn increases the load on the rest of the elements and the process continues until the entire structure collapses.

This process has a powerful analogue in organisations. A positive feedback loop is sometimes triggered if a group of dissatisfied experts leaves the organisation because of excessive work load (Factor $A$ ). If no sufficient appointments are made because of illconceived management strategy, the duties of people who have left are distributed across the remaining people. The increased work load (factor $B$ ) on the remaining staff causes further dissatisfaction (Factor $A$ ) and more experts leave. This self-reinforcing process may continue until the organisation is depleted of high-quality staff and the service the organisation provides deteriorates beyond repair.

In human behaviour, frequent positive feedback loops with profound negative impact are created by the strongly interacting factors _bdief' and _choice'. For example, belief in the incorrect Weibull model for determining the probability of fracture of components initiated by flaws determined its choice as a model for many decades. The choice of the incorrect Weibull model then reinforced the belief in this model to the extent of ignoring experimental data sets clearly contradicting the strictly increasing Weibull function. As a result, the interaction of belief $=$ and choice' led to a firmly entrenched false modelling paradigm, blind to the mounting contradicting evidence from experiments, computer simulations and theoretical arguments.

In the economy, the withdrawal of investment from a country, causes poverty and poverty leads to a further withdrawal of investment.

\subsubsection{Case study: Growth of damage sustained by a positive feedback loop with negative impact}


In addition to providing the conditions for a rapid growth of a beneficial outcome, the positive feedback loop often works in the opposite direction and provides the conditions for a rapid growth of damage.

Suppose that at time $t$, the rate of increasing the damage $y$ is positive and proportional to the existing damage $y$. Initially, at time $t=0$, the damage is equal to $y_{0}$. At time $\Delta t$, the damage $y$ will increase to $y_{1}=y_{0}+\Delta y_{0}>y_{0}$. At time $2 \Delta t$, the already increased damage $y_{1}$ will give rise to even larger increase $\Delta y_{1} \quad\left(\Delta y_{1}>\Delta y_{0}\right)$ and the damage becomes $y_{2}=y_{1}+\Delta y_{1}>y_{1}$, etc. The growth of damage is sustained by a positive feedback loop because the more damage $y$ is produced the more further damage is generated.

It needs to be pointed out that the growth of damage sustained by a positive feedback loop is not necessarily an exponential growth.

Assume that the rate of increase $d y / d t$ of the damage $y$ is proportional to the existing damage $y$ raised into some power $1+\varepsilon>0$, where $\varepsilon \neq 0, k$ is some positive constant $(k>0)$ and $t$ is the time:

$$
d y / d t=k y^{1+\varepsilon}
$$

If the initial damage $y$ is equal to $y_{0}$ (at $t=0$ ), separating the variables of equation (9) yields $d y / y^{1+\varepsilon}=k d t$ which, after integration and determining the integration constant, yields the growth law

$$
y=\frac{1}{\left(\frac{1}{y_{0}^{\varepsilon}}-k \varepsilon t\right)^{1 / \varepsilon}}
$$

As can be seen, if $\varepsilon>0$ the damage growth sustained by the positive feedback loop is extremely rapid and after a finite time $t=\frac{1}{y_{0}^{\varepsilon} k \varepsilon}, y \rightarrow \infty$.

At the beginning, the damage growth is slow, which can give a wrong perception of safety. With time, what appeared to be a minor problem, very quickly transforms into a major problem. By the time the late stages of damage growth are reached, it may be too late to do anything to limit the extent of damage.

An example of damage growth by a positive feedback loop can be given with the growth of fatigue cracks. The fatigue crack growth is given by the Paris law (Paris et al, 1961; Paris and Erdogan, 1963)

$$
d a / d t=k a^{m / 2}
$$

where $\underline{a}^{6}$ is the current length of the fatigue crack, $k$ is a constant depending on the geometry, the material and the loading stress range and $m$ is a material constant (the Paris exponent). The initial size of the crack (at time $t=0$ ) is $a_{0}$.

For typical values of the Paris exponent, the constant $m$ is greater than two $(m>2)$. Consequently, setting $\varepsilon=m / 2-1$ gives

$$
d a / d t=k a^{1+\varepsilon}
$$

which is essentially equation (9). Its integration yields

$$
a=\frac{1}{\left(\frac{1}{a_{0}^{\varepsilon}}-k \varepsilon t\right)^{1 / \varepsilon}}
$$

which describes the length of the crack with time. Initially, the fatigue crack growth sustained by the positive feedback loop is slow but it accelerates with increasing the length of the 
crack. If the crack length that triggers fast fracture is $a_{f}$, the rate of the fatigue crack growth increases as the crack length approaches this value. With increasing time, the rate of damage accumulation $d a / d t$ increases (Figure 14).

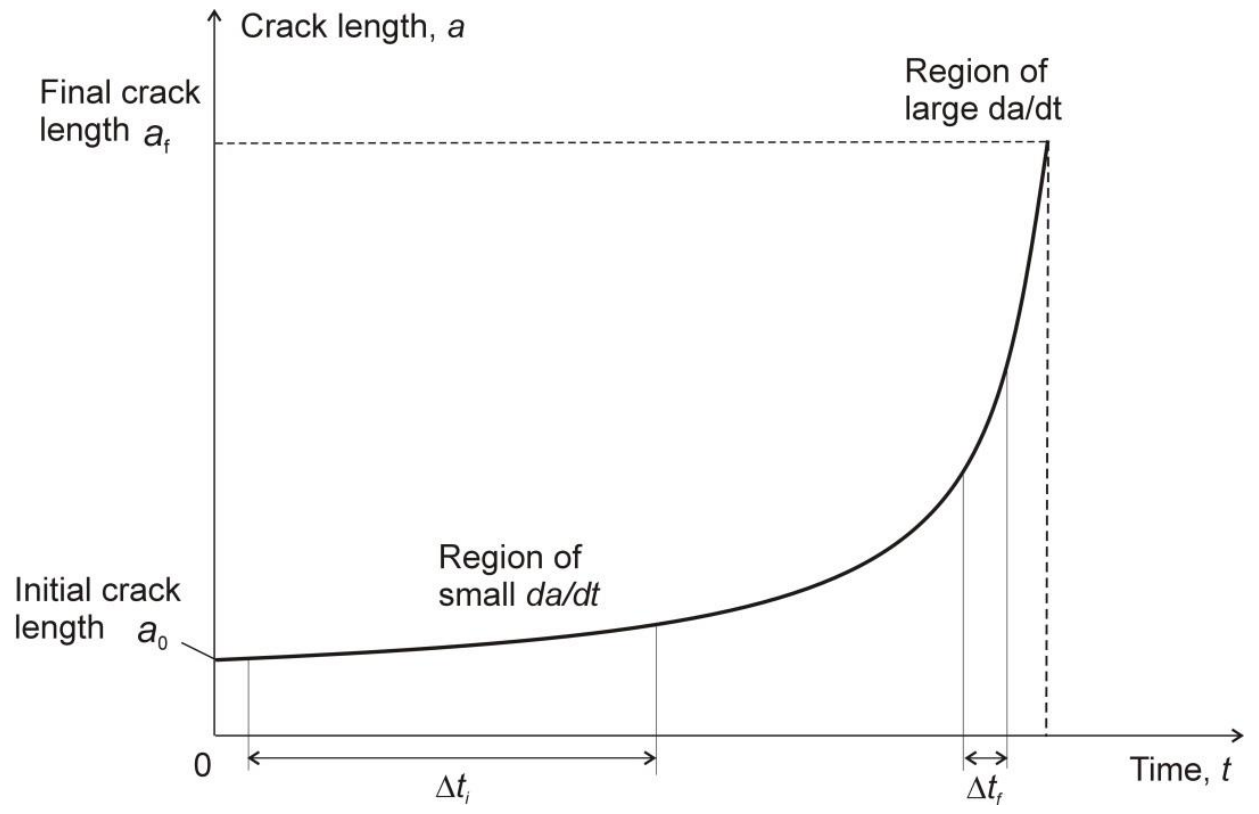

Figure 14. In a positive feedback loop, the rate of damage accumulation increases with time.

During damage growth sustained by a positive feedback loop, the frequency of the damage monitoring intervals should increase as damage progresses. The length of the damage inspection intervals $\Delta t_{i}$, in the initial stages of damage accumulation, is significantly larger than the length $\Delta t_{f}$ of the damage inspection intervals in the final stages of damage accumulation (Figure 14).

The growth of damage caused by a positive feedback loop can be reduced by inhibiting the positive feedback loop so that the damage accumulation is slowed down significantly.

Suppose that an implemented inhibition measure causes the rate of accumulation of damage to be proportional to the square root of the damage $y$. The constant $\varepsilon$ in equation (9) is then $\varepsilon=-1 / 2$. Substituting $\varepsilon=-1 / 2$ in the solution (10) yields the polynomial growth law

$$
y=\left(\sqrt{y_{0}}+\frac{k t}{2}\right)^{2}
$$

which describes the damage accumulation with time. The damage growth described by equation (14) corresponding to $\varepsilon=-1 / 2$, is significantly slower than the damage growth corresponding to $\varepsilon>0$ given by equation (10).

Even in the case where the constant $\varepsilon$ is reduced to zero $(\varepsilon=0)$, the integration of equation (9) yields the exponential growth:

$$
y=y_{0} e^{k t}
$$

which is slower than the damage growth given by equation (10), where $\varepsilon>0$.

The exponential growth described by equation (15) is the growth of capital due to continuous compounding. 


\subsection{Self-reinforcement by creating positive feedback loops with positive impact}

Positive feedback loop with positive impact is a self-reinforcing loop which promotes and sustains growth.

A positive feedback loop with positive impact is present in the important case where an initial investment $A$ in reliability improvement leads to a more superior product $B$ which in turn leads to increased sales $C$ and the increased sales $C$ lead to increased investment $A$ in the reliability improvement. The dynamics of this process has been illustrated in Figure 13b. The result is a sustained growth towards a superior product characterised by increased reliability and reduced risk of failure.

In capturing the benefits from positive feedback loops with positive impact, accelerators can be used.

In business, positive feedback loops are powerful engines of growth. Business creates value, which attracts customers. Customers bring profits that are reinvested in the business which leads to more value creation. Surveys can be used as accelerators of this positive feedback loop. Comments from customers in the surveys are used to tailor the content of the business to the customers' expectations, which leads to improved services, expanding the business and further growth.

Positive feedback loops can also be used in bistable systems to guarantee fast transition into a more reliable state. If the input is slightly higher than the equilibrium state, the positive feedback causes the output to move in the same direction, until the output reaches the nearest upper equilibrium state. If the input is slightly lower than the equilibrium state, the positive feedback causes the output to move fast in the same direction until it reaches the nearest lower equilibrium state. Once an equilibrium state has been reached, the system remains in that state. The larger the deviation of the output from the specified level, the larger is the input correction increasing the deviation of the output in the same direction. In electrical switches, for example, the positive feedback control minimises the length of arcing during switching thereby minimising the wear out and extending the life of the switches.

Positive feedback loops are also used in circuits which trigger a particular action if a signal crosses a pre-determined threshold value. The Schmitt trigger circuit, for example, uses a positive feedback control to force the output in one of the two available stable logic states.

\subsubsection{Case study: Positive feedback loop providing self-reinforcement by self-energizing}

Positive feedback loops can also be used with success for improving the reliability of a system and reducing the risk of failure. The self-energizing friction holder which supports the weight $G$ of a sheet in Figure 15 is an example of self-reinforcement through a positive feedback loop. Once the friction pads of the holder touch the sheet, a small normal force $N$ appears and excites a proportional friction force $F_{f r}=\mu N$ where $\mu$ is the coefficient of friction between the friction pad and the sheet of material. The friction force $F_{f r}=\mu N$ causes rotation of the link OP around the pin O. However, the geometric constraint causes the normal force $N$ to increase which in turn increases the friction force $F_{f r}=\mu N$ and so on. This process of self-excitation continues until the friction forces $F_{f r}=\mu N$ acting on both sides of the sheet counterbalance the weight $G$ of the sheet.

As result of the positive feedback loop, the friction forces $F_{f r}=\mu N$ quickly reach large magnitudes despite that the friction pads were initially energized with a small load. The result is a reliable support of the weight $G$ of the sheet. 
The smallest coefficient of friction $\mu^{*}$ necessary to achieve self-energizing can be determined from the equilibrium condition of the link OP. From the equilibrium condition of the moments with respect to point $O$ :

$$
N c-\mu N a=0
$$

which is equivalent to $c-\mu a=0$. Self-energising is present if $c \leq \mu a$. The minimum coefficient of friction for a self-energising is given by $\mu^{*}=c / a$. Thus, for $c=15 \mathrm{~mm}$ and $a=60 \mathrm{~mm}, \mu^{*}=15 / 60=0.25$.

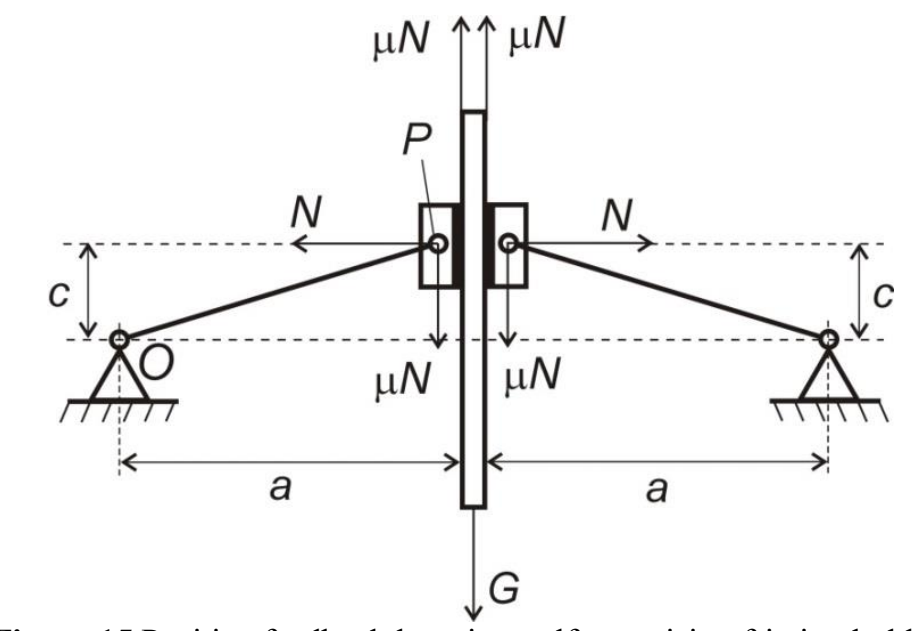

Figure 15 Positive feedback loop in a self-energising friction holder.

\section{Application and future work}

The method of _self-reinforcement" presented in this paper is a domain-independent method for reliability improvement and risk reduction. It transcends the area of mechanical engineering where it originated and can be applied in diverse areas of human activity. The application of this method covers mechanical engineering, civil engineering and construction, electronics, software engineering, chemical engineering, financial control, management, project management, environmental sciences, logistics supply, economics, etc. The method of self-reinforcement for risk reduction does not rely on reliability data or knowledge of physical mechanisms underlying possible failure modes. As a result, it is very well suited for new designs, with no failure history and unknown failure mechanisms.

The method of self-reinforcement often improves reliability at no extra cost or at a low cost. In this respect, it differs from many traditional methods for improving reliability (e.g. introducing reinforecement, upgrading components, introducing redundancy, condition monitoring) which are all associated with substantial investment. Without methodological knowledge of the method of self-reinforcement, opportunities for decreasing risk will not be recognised and will be missed.

By providing the foundation for improving reliability and reducing risk in diverse areas of human activity, the method of self-reinforcement contributes to changing the existing risk reduction paradigm based exclusively on domain-specific methods. However, the method of self-reinforcement is not a substitute for domain-specific methods for improving the reliability of engineering products. It rather serves as a powerful enhancement of the domainspecific reliability improvement and risk reduction.

This method is part of a future powerful framework of domain-independent methods for reliability improvement and risk reduction. The impact of such a framework is significant 
because it will enhance the reliability of products and operations for any company and organisation. To any company, reliability is one of the most important attributes of its products which translates into competitive advantage and secure market position.

A framework of domain-independent methods for risk reduction will also provide the basis for strong knowledge exchange with various industries and for strong interdisciplinary research. Researchers and engineers, after receiving training in domain-independent risk reduction methods will be able to solve complex reliability improvement problems in their specific industries/domains. In turn, the problems encountered in the specific industry/domain will stimulate the development of the domain-independent reliability improvement and risk reduction methods. This creates a positive self-reinforcing feedback loop which benefits both industry and science.

\section{CONCLUSIONS}

1. The method of self-reinforcement has been introduced as an important domainindependent method for improving reliability and reducing risk. An important feature of selfreinforcement is that increasing the external/internal forces intensifies the system's response against these forces. As a result, the driving net force towards precipitating failure is decreased.

2. Two principal ways of self-reinforcement have been identified: reinforcement by capturing a proportional compensating factor and reinforcement by using feedback loops.

3. Mechanisms of transforming forces and motion into a self-reinforcing response have been introduced and demonstrated through appropriate examples.

4. Mechanisms achieving self-reinforcement by self-aligning and modified geometry have been introduced.

5. The potential of positive feedback loops for achieving self-reinforcement and risk reduction has been demonstrated. The rapid growth/decay, the fast transition of the system into a more stable state and the self-energising provided by positive feedback loops can be used successfully for reliability improvement and risk reduction.

6. It was demonstrated that eliminating or inhibiting positive feedback loops with negative impact is an important risk reduction resource.

7. A classification was proposed of methods and techniques for reliability improvement and risk reduction based on the method of self-reinforcement.

8. The method of self-reinforcement often improves reliability and reduces risk at no extra cost. It is a part of a future powerful framework of domain-independent methods for risk reduction which transcends mechanical engineering and can be applied in diverse areas of human activity. 


\section{REFERENCES}

Altshuller, G. S. (1984). Creativity as an exact science: The theory of the solution of inventive problems. New York: Gordon and Breach Science Publishing.

Altshuller, G. S. (1996). And suddenly the inventor appeared, TRIZ, the theory of inventive problem solving, Translation from Russian by Lev Shulyak. Worcester, MA: Technical Innovation Center.

Altshuller, G. S. (1999). The innovation algorithm, TRIZ, systematic innovation and technical creativity. Worcester: Technical Innovation Center, Inc.

Bergman B., J. de Mare, S. Loren and T. Svensson, editors, Robust design methodology for reliability, Edited by, Willey, Chichester UK, (2009).

Bird, G. C., \& Saynor, D. (1984). The effect of peening shot size on the performance of carbon-steel springs. Journal of Mechanical Working Technology, 10(2), 175-185. doi:10.1016/0378-3804(84)90065-2.

Collins, J. A. (2003). Mechanical design of machine elements and machines. New York: John Wiley \& Sons, Inc.

Costache, A., Glejbøl, K., Sivebæk, I. M., \& Berggreen, C. (2016). Improved Friction Joint With Self-Locking Grips. Journal of Offshore Mechanics and Arctic Engineering, 38(5).

Dowling N.E. (1999), Mechanical behavior of materials, 2nd ed., Prentice Hall.

Feng X., Ouyang M., Liu X., Lu L., Xia Y., He X., Thermal runaway mechanism of lithium ion battery for electric vehicles: A review, Energy Storage Materials, http://dx.doi.org/10.1016/j.ensm.2017.05.013.

French M., Conceptual design for engineers, 3rd ed., Springer-Verlag London Ltd, London (1999).

Fu, Y., Ge, E., Su, H., Xu, J., \& Li, R. (2015). Cold expansion technology of connection holes in aircraft structures: A review and prospect. Chinese Journal of Aeronautics, 28(4), 961-973. doi:10.1016/j.cja.2015.05.006.

Gadd K. (2011). Triz for engineers: Enabling inventive problem solving, Wiley.

Kelly P.M., \& Francis-Rose L.R. (2002). Progress in Materials Science. The Martensitic Transformation in Ceramics - its role in transformation toughening, 47(5), 463-557.

Lindroos, M., Apostol, M., Heino, V., Valtonen, K., Laukkanen, A., Holmberg, K., \& Kuokkala, V. T. (2015). The deformation, strain hardening, and wear behaviour of chromium-alloyed hadfield steel in abrasive and impact conditions. Tribology Letters, 57(3), 24. doi:10.1007/s11249-015-0477-6.

Mattheck, C. (1998). Design in nature. Berlin: Springer Verlag. 
Matthews C. (1998). Case studies in engineering design. London, Arnold.

Meriam J.L, Kraige L.G., Bolton J.N. (2016), Engineering mechanics: Statics, 8th ed., Wiley.

MIL-STD-1629A, US Department of Defence procedure for performing a failure mode and effects analysis. (1977). Washington, DC: US Department of Defence.

Niku-Lari, A. (1981, September 14-17). Shot-peening, in the first international conference on shot peening, Paris. Oxford: Pergamon Press.

Orloff, M. (2006). Inventive thinking through TRIZ (2nd ed.). Springer.

Pahl, G., Beitz, W., Feldhusen, J., \& Grote, K. H. (2007). Engineering design. Berlin: Springer. doi:10.1007/978-1-84628-319-2.

Paris, P. C., \& Erdogan, F. (1963). A critical analysis of crack propagation laws. Journal of Basic Engineering, 85(4), 528-534. doi:10.1115/1.3656900

Paris, P. C., Gomez, M. P., \& Anderson, W. P. (1961). A rational analytic theory of fatigue. The Trend in Engineering, 13, 9-14.

Rantanen K., Domb E. (2008). Simplified TRIZ, 2nd edition, Auerbach Publications.

Savransky S.D. (2000). Introduction to TRIZ methodology of inventive problem solving, CRC press LLC.

Terninko J., A.Zusman, B.Zlotin (1998). Systematic Innovation: An introduction to TRIZ, CRC Press LLC.

Uicker J.J. Jr, G.R. Pennock and J.E. Shigley. (2003), Theory of machines and mechanisms, 3rd ed., Oxford University Press, New York (2003).

Zhang, L., Konyukhov, A., Mok, E., \& Choo, H. (2013). Increasing the road safety of e-bike: design of protecting shell based on stability criteria during severe road accidents, Proceedings of the 2013, International conference on electrical, control and automation engineering $\left(\mathrm{ECAE}_{=}{ }^{13}\right)$ (pp. 485-489). 